\title{
ANTIOXIDANTS THE POWERFUL NEW WEAPONS IN THE FIGHT AGAINST PERIODONTAL DISEASES
}

\author{
Baljeet Singh ${ }^{1}$, Shivani Bhickta ${ }^{2}$, Rajesh Gupta ${ }^{3}$, Sachin Goyal $^{4}$, Ram Rattan Gupta ${ }^{5}$ \\ ${ }^{1}$ Prof. \& Head, Department of Periodontology, Bhojia Dental College and Hospital, Distt. Solan, Himachal Pradesh (India) \\ ${ }^{2}$ PG Student, Department of Periodontology, Bhojia Dental College and Hospital, Distt. Solan, Himachal Pradesh (India) \\ ${ }^{3}$ PG Student, Department of Periodontology, Bhojia Dental College and Hospital, Distt. Solan, Himachal Pradesh (India) \\ ${ }^{4}$ Reader, Department of Periodontology, Bhojia Dental College and Hospital, Distt. Solan, Himachal Pradesh (India) \\ ${ }^{5}$ Reader Department of Periodontology, Bhojia Dental College and Hospital, Distt. Solan, Himachal Pradesh (India) \\ Corresponding Author: \\ ${ }^{1}$ Mobile: 919814510711 Email: baljeet065_singh@yahoo.co.in
}

\begin{tabular}{|l|}
\hline Received : \\
$25^{\text {th }}$ March, 2013 \\
Accepted: \\
$1^{\text {st }}$ June, 2013 \\
Available online: \\
$25^{\text {th }}$ August, 2013 \\
\hline
\end{tabular}

\section{INTRODUCTION}

It is believed that while the primary etiological agent in periodontal diseases is bacteria within the subgingival biofilm, the majority of periodontal tissue destruction is caused by an inappropriate host response to those microorganisms and their products. More specifically, a loss of homeostatic balance between proteolytic enzymes (e.g. neutrophil elastase) and their inhibitors (e.g. $\alpha 1$-antitrypsin), and reactive oxygen species (ROS) and the antioxidant defense systems that protect and repair vital tissue, cell, and molecular components is believed to be responsible. Free radicals have been defined as any species capable of independent existence that contain one or more unpaired electrons. They are, by nature, highly reactive and diverse species, capable of extracting electrons and thereby oxidizing a variety of biomolecules vital to cell and tissue function. These, not only include oxygen free radicals, but also nitrogen and chlorine species. ${ }^{1}$ The reactive species superoxide $\left(\mathrm{O}_{2}{ }^{-}\right)$, hydrogen peroxide $\left(\mathrm{H}_{2} \mathrm{O}_{2}\right)$, hydroxyl radical $\left(\mathrm{HO}^{-}\right)$, nitrogen oxide $\left(\mathrm{NO}^{-}\right)$, peroxynitrite $\left(\mathrm{ONOO}^{-}\right)$and hypochlorous acid $(\mathrm{HOCl})$, are all products of normal metabolic pathways of the human organs, but under certain

\section{ABSTRACT}

The human inflammatory periodontal diseases are amongst the most common of chronic diseases. The eminant inflammatory cell $(96 \%)$ within the healthy connective tissue and epithelium of the gingiva is activate PMNLs leading to an increased production of reactive oxygen species (ROS). Chronic inflammation (he nearby cells to elevated levels of free radicals (ROS) due to extracellular release from phagocytic cells. Antioxidants block the process of oxidation by neutralizing free radicals. In doing so, the antioxidant themselves become oxidized. Because of this, there is a constant need to replenish our antioxidant resources.

Keywords: Antioxidants, Periodontal Diseases, Reactive oxygen species.

conditions, when in excess they can exert harmful compounds. In normal physiology there is a dynamic equilibrium between ROS activity and antioxidant defense capacity and when that equilibrium shifts in favour of ROS, either by a reduction in antioxidant defences or an increase in ROS production or activity, oxidative stress results. ${ }^{2}$

Oxidative stress was defined by Sies, 1991 as a disturbance in the pro-oxidant-antioxidant balance in favour of the former, leading to potential damage (Figure 1). It is estimated that between 1 billion and 3 billion ROS are generated per cell per day.

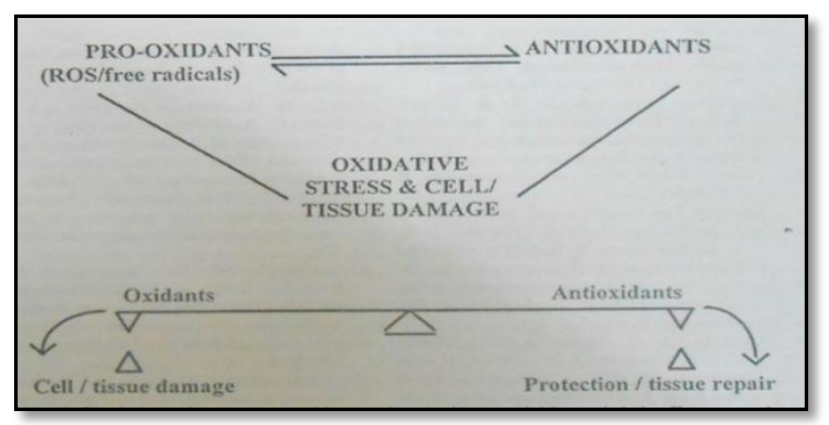

Figure 1

Significant ROS generation by neutrophils requires a minimum oxygen tension of about $1 \%$ and a $\mathrm{pH}$ of 
7.0-7.5. Both these conditions are found within periodontal pockets, indicating that chronic or excess ROS production is possible at this important site of periodontal tissue damage. Neutrophils have an ability to withstand the hostile environment of the periodontal pocket. Thus, neutrophils are able to function and initiate respiratory burst activity in the presence of sulfide at the toxic levels found in diseased sites.

- Fibroblasts, which represent the largest population of cells in healthy gingiva and PDL, are able to spontaneously release detectable levels of ROS in culture media containing $\mathrm{Ca}^{2+}$ (Murrell et al 1990)

\section{ROS CAUSE PERIODONTAL TISSUE DAMAGE BY}

- Ground substance degradation

- Collagenolysis either directly or as a result of oxidation of antiproteases

- Stimulation of excessive pro-inflammatory cytokine release through NF-kb activation.

- $\mathrm{PGE}_{2}$ production via lipid peroxidation.

Antioxidants are a group of substances which, when present at low concentrations, in relation to oxidizable substrates, significantly inhibit or delay oxidative processes, while often being oxidized themselves. Epidemiological studies demonstrate that a higher intake of fruits and vegetables (FV) is associated with a lowered risk of atherogenic cardiovascular disease (Joshipura et al. 1999), ischaemic stroke (Joshipura et al. 2001) and mortality (Knekt et al. 1996, Sauvaget et al. 2003). Diets rich in vegetables and Vitamin-C also appear to associate positively with better periodontal health and negatively with periodontal disease progressing more rapidly in undernourished populations (Enwonwu et al. 2002). ${ }^{3}$

\section{CLASSIFICATIONS OF ANTIOXIDANTS ${ }^{1}$}

Antioxidants can be categorized by several methods: (Chapple and Mathews 2007)

1. their mode of function;

2. their location of action (intracellular, cell membrane or extracellular);

3. solubility (lipid or water), although considerable interaction exists between aqueous and lipophilic antioxidants in protecting lipoproteins against oxidative damage;

4. their structural dependents;

5. their origin/source, e.g. dietary or nondietary sources.

\section{Antioxidants Classified by Mode of Action}

a. Preventative antioxidants:

- Enzymes (that suppress the formation of free radical): superoxide dismutase enzymes, catalase, glutathione peroxidase, DNA repair enzymes, e.g. poly (ADP-ribose) polymerase, etc.

- Metal ion sequestrators: albumin, lactoferrin, transferrin, carotenoids, superoxide dismutase, catalase, glutathione peroxidase, glutathione reductase, uric acid, polyphenolic flavenoids etc.

\section{b. Scavenging (chain breaking) antioxidants}

- Ascorbate (vitamin C), carotenoids (including retinol - vitamin A), uric acid, $\alpha$-tocopherol (vitamin E), polyphenols (flavenoids), bilirubin, albumin, glutathione and other thiols (free or protein bound) etc.

\section{Examples of Key Antioxidants Classified by} Location

- Intracellular: Superoxide dismutase enzymes 1 and 2, catalase, glutathione peroxidase, DNA repair enzymes e.g. poly (ADP-ribose) polymerase etc.

- Extracellular: Superoxide dismutase enzyme 3, selenium-glutathione peroxidase, reduced glutathione, lactoferrin, albumin, ascorbate, carotenoids, uric acid etc.

- Membrane associated: $\alpha$-Tocopherol.

\section{Key Antioxidants Classified by Solubility}

- Water soluble: Haptoglobin, albumin, ascorbate, uric acid, polyphenolic flavenoids, reduced glutathione and other thiols, cysteine etc.

- Lipid soluble: $\alpha$-Tocopherol, carotenoids, bilirubin, quinones (e.g. reduced ubiquinone). 


\section{Antioxidants Classified by Structures They Protect}

a) DNA protective antioxidants

- Superoxide dismutase enzymes 1 and 2, glutathione peroxidase, DNA repair enzymes [e.g. poly (ADP-ribose) polymerase], reduced glutathione, cysteine.

b) Protein-protective antioxidants

- Sequestration of transition metals by preventative antioxidants (Uric acid).

- Scavenging by competing substrates (vitC).

- Antioxidant enzymes (superoxide dismutase, catalase, glutathione peroxidase).

\section{c) Lipid-protective antioxidants}

$\alpha$-Tocopherol (vitamin E), ascorbate (vitamin C), carotenoids (including retinol - vitaminA), reduced ubiquinone, reduced glutathione, glutathione peroxidase, bilirubin.

\section{Some Key Antioxidants Classified by Their Origin}

a) Exogenous antioxidants (obtained only though the diet):

- Phytonutrients, Carotenoids, ascorbic acid, tocopherols, polyphenols (e.g. flavenoids, catechins such as epigallocatechin-gallate), folic acid, cysteine.

b) Endogenous antioxidants (synthesized by the body):

- Catalase, superoxide dismutase, glutathione peroxidase, glutathione-S-transferase, reduced glutathione, ceruloplasmin, transferrin, ferritin, glycosylases, peroxisomes, proteases.

\section{c) Synthetic:}

- $\mathrm{N}$-acetylcysteine, penicillinamine, tetracyclines.

\section{MECHANISM OF ACTION ${ }^{2,4}$}

Two principle mechanisms of action have been proposed for antioxidants by Ingold KU in 1968. The first is a chain-breaking mechanism, by which the primary antioxidant donates an electron to the free radical present in the system (e.g., lipid radical).

The second mechanism involves removal of ROS/RNS initiators (secondary antioxidants) by quenching chain-initiating catalysts.

1. Electron Donation ( as a reducing agent)

2. Metal Chelation (thereby eliminating potential free radicals)

3. Co-Antioxidants (synergistic antioxidant effect)

4. Gene Expression Regulation

\section{PREDOMINANT ANTIOXIDANTS}

Ascorbic Acid (Vit-C) ${ }^{1,5}$

Vitamin $\mathrm{C}$ or L-ascorbic acid is water-soluble and is present in its deprotonated state under most physiologic conditions. Commercially available as Limcee, C-VIT.

It is considered to be the most important antioxidant in extracellular fluids.

Its activities may be summarized as:

- scavenging water-soluble peroxyl radicals;

- scavenging superoxide and perhydroxyl radicals;

- prevention of damage mediated by hydroxyl radicals on uric acid;

- scavenger of hypochlorous acid;

- decreases heme breakdown and subsequent $\mathrm{Fe}^{2+}$ release thereby preventing Fenton reactions;

- scavenger of singlet oxygen and hydroxyl radicals

- re-forms $\alpha$-tocopherol from its radical;

- protects against ROS-release from cigarette smoke

- acts as a strong antioxidant in the plasma and presents a synergistic effect with other antioxidants (co-antioxidant).

\section{$\alpha$-Tocopherol ${ }^{1,6}$}

Vitamin E is generally regarded as the most important and effective lipid soluble anti-oxidant in- 
vivo. It is vital in maintaining cell membrane integrity against lipid peroxidation by peroxyl radical scavenging. Its antioxidant behavior is the result of a single phenolic $\mathrm{OH}$ group, which when oxidized gives rise to vitamin $\mathrm{E}$ radical. Commercially available as EVION-LC, ELIMCEE.

Vitamin E possesses anti-inflammatory as well as antioxidant properties (Brock, 2005), which are as follows:

- Inhibition of protein kinase $\mathrm{C}$ and subsequent platelet aggregation;

- Inhibition of nitric oxide production by vascular endothelium;

- Inhibition of superoxide production by macrophages and neutrophils (Azzi 2002), by inhibition of $\mathrm{p} 47 \mathrm{phox}$ phosphorylation during NADPH oxidase activation.

\section{CAROTENOIDS}

Carotenoids ( $\alpha$ - and $\beta$-carotene, lycopene, lutein astaxanthin, canthaxanthin, retinol etc.) are lipophilic and higher plasma concentrations have been shown to protect against various inflammatory and malignant diseases. Derived only from the diet (green vegetables, tomatoes, fruits), lycopene predominates in plasma, with tomatoes being the main dietary source in humans (other sources include red grapefruits and water melon).

Lycopene, the carotenoid that gives ripe tomato its bright red colour, is an effective natural antioxidant and a quencher of free radicals. Lycopene exhibits the highest physical quenching rate with singlet oxygen $^{7}$ and is at least three-fold more effective than $\beta$-carotene in preventing cell death by quenching nitrogen oxide $\left(\mathrm{NOO}^{-}\right)$radicals. It also reverses DNA damage induced by $\mathrm{H}_{2} \mathrm{O}_{2}{ }^{8}$ A recent study investigated the relationship between monthly tomato consumption and serum lycopene levels, and a selfreported history of congestive heart failure $(\mathrm{CHF})$ in individuals with periodontitis, and concluded that a relationship exists between periodontitis and CHF risk, and high monthly tomato consumption appears to affect this relationship in a positive direction in periodontitis subjects. . Commercially available as LycoRed, G-Power Lyco.

\section{POLYPHENOLS (POLYPHENOLIC FLAVENOIDS) $)^{1,10}$}

Polyphenols are absorbed following dietary intake of vegetables, red wine and tea. The cellular mechanisms involved in free radicals (FR)/ reactive oxygen species (ROS) protection is mainly due to their direct antioxidant properties (e.g. by sparing vitamin $\mathrm{E}$ or by regenerating vitamin $\mathrm{C}$ ) or to their inhibitory activity towards lipooxygenase.

Polyphenols function by:

- Radical scavenging;

- Terminating lipid peroxidation;

- iron chelation;

- sparing vitamin E;

- restoration of vitamin $\mathrm{C}$.

Glutathione $^{1,11}$

Glutathione (GSH) is an essential tripeptide with many important functions. Glutathione's three major roles in the body are that it acts as an intrinsic antioxidant, immune booster and detoxifier.

Glutathione exists in oxidized (GSSG) and reduced (GSH) forms and GSH is a ubiquitous thiol that plays a major role in human physiology and pathology, for several reasons:

- It is one of the most vital intracellular antioxidant scavengers;

- It is essential to the glutathione peroxidase antioxidant enzyme system, which removes hydrogen peroxide by converting two GSH molecules to one GSSG molecule and water;

- It plays a major role in maintaining the intracellular redox balance and thus regulating signaling pathways which are affected by oxidative stress

\section{URIC ACID ${ }^{1,12}$}

Uric acid is a weak acid distributed throughout the extracellular fluid as sodium urate. Uric acid is produced from purines by the enzyme xanthine oxidase via the purine metabolism pathway (Warrin et al., 2000). 
Its antioxidant activities include:

- scavenger of singlet oxygen;

- scavenger of hydroxyl radicals;

- scavenger of hypochlorous acid;

- protection of $\alpha 1$-antitrypsin when combined with ascorbate;

- binding of divalent metal ions preventing Fenton chemistry.

\section{ANTIOXIDANTS IN PERIODONTAL HEALTH}

Low levels of most antioxidants are a risk factor for periodontal disease and infection. Free radicals are released as a result of bacteria clearance and killing. Periodontal tissue depends on natural antioxidants to overcome this oxidative stress and maintain homeostasis. When antioxidants are depleted, the ability of gingival tissue to overcome oxidative stress, maintain normal tissue, and control the bacterial damage appears to be compromised.

Grossi's (1999) et al examined serum levels of antioxidant nutrients and their relationship to periodontal disease. Results showed that selenium has the strongest association with gum disease, with low levels increasing the risk by 13 -fold. Low levels of vitamins $A$ and $C, \alpha$-carotene, and $\beta$-crytoxanathin also increased the risk. ${ }^{13}$

Tinkler et al (1994) studied the quenching of singlet oxygen by $\beta$-carotene, astaxanthin, lycopene, and canthaxanthin bound to the surface of lymphoid cells and found that all 4 carotenoids protected the cells against the photodynamic reaction sensitized by rose bengal or meso-tetra (4-sulphonatophenyl) porphine, the highest protection being given by lycopene. It has also been shown that $\beta$-carotene and lycopene are effective protectors of lymphocyte cells from nitrogen dioxide radical damage, but lycopene is at least twice as effective as $\beta$-carotene (Bohm et al.,1995). ${ }^{14}$

Panjamurthy et al. (2005) found lower plasma vitamin $\mathrm{C}$, vitamin $\mathrm{E}$, and $\mathrm{GSH}$ in periodontitis patients even after adjusting for protein levels, whereas antioxidant enzyme levels were raised, the authors attributing this to a protective response to oxidative stress (thiobarbituric acid reactive substance levels were raised in periodontitis subjects). ${ }^{15}$
Sobaniec $\mathrm{H}$ et al (2007) estimated the activity of chosen antioxidants in blood serum and saliva in patients with periodontal disease treated due to epilepsy. Twenty-five epileptics and fifteen control persons were involved in the study. The activity of selected endogenous antioxidants were determined by spectrophotometric assay. Concentrations of vitamin $\mathrm{A}$ and vitamin $\mathrm{E}$ were measured using liquid chromatography. The analysis of the serum and saliva from patients with overgrown gingiva revealed: reduced activity of superoxide dismutase, glutathione peroxidase and glutathione reductase, elevated lipid peroxides, and decreased concentration of ascorbic acid and $\alpha$-tocopherol. All values were statistically significant. Results indicated that oxidant-antioxidant disturbances in epileptic patients, play an important role in the pathomechanism of periodontal disease. $^{16}$

Akman S et al., (2013) aimed to investigate the role of alpha lipoic acid (ALA) and Vit-C substances in the treatment of alveolar bone resorption in periodontal diseases. Their study clearly demonstrated that oxidative stress-mediated RANKL cell activity plays an important role in alveolar bone resorption in the rat model with experimental periodontitis. ALA and Vit-C provide beneficial improvements in osteoclast-mediated bone resorption and encourage a reduction in bone alkaline phosphatase (B-ALP) activation. The results of their study support the concept that the systemic administration of ALA and ALA + Vit-C could inhibit inflammatory bone resorption and osteoclast activation, which reveals a new, possible method for preventing periodontal bone resorption and improving periodontal health. ${ }^{17}$

Trivedi S et al., (2013) investigated the impact of diabetes, a known risk factor for periodontitis, on antioxidant enzymes superoxide dismutase (SOD), glutathione reductase (GR), catalase (CAT) activities and free radical damage marker malondialdehyde (MDA) levels in blood and saliva of individuals with chronic periodontitis (CP). Their study gives a convincing support in favor of the etiological role of free radical injury in periodontitis with diabetes. It shows that the compensatory mechanism of the body is partially collapsed due to excessive production of free radicals during periodontitis, and is not able to 
cope with increased free radical generation due to diabetes, thereby worsening the situation. ${ }^{18}$

\section{CONCLUSION}

The imbalance between reactive oxygen species and antioxidant defense systems may increase the oxidative burden and lead to the damage of macromolecules. Such processes are thought to play a role in pathological processes of various diseases, including periodontal diseases. There is increasing evidence from epidemiological studies, animal experiments, and in-vitro investigations that an increased intake of antioxidants is associated with a diminished risk for several diseases.

\section{REFERENCES}

1. Chapple ILC, Mathews JB. The role of reactive oxygen and antioxidant species in periodontal tissue destruction. Perio 2000. 2007; 43:160-232.

2. Vaya J, Aviram M. Nutritional Antioxidants: Mechanisms of Action, Analyses of Activities and Medical Applications. Current Medicinal Chemistry-Immunology, Endocrine \& Metabolic. May 2001;1(1):99.

3. Chapple ILC, Milward MR, Ling-Mountford N, Weston P, Carter K, Askey K, Dallal GE, De Spirt S, Sies H, Patel D, Matthews JB. Adjunctive daily supplementation with encapsulated fruit, vegetable and berry juice powder concentrates and clinical periodontal outcomes: a doubleblind RCT. J Clin Periodontol 2012;39:62-72.

4. Manjunath RGS. Role of Antioxidants as an adjunct in periodontal therapy. J. Academy Adv Dental Research 2011;2(2):9-16

5. Sies $\mathrm{H}$ and Stahl W. Vitamins E and C, $\alpha$-carotene, and other carotenoids as antioxidants Am J Clin Nutr 1995;62 (suppl): 1315S-1321S.

6. Gutteridge JMC, Halliwell B. Antioxidants in nutrition, health and disease. New York: Oxford University Press, 1994.
7. Di Mascio P, Kaiser S, Sies H. Lycopene as the Most Efficient Biological Carotenoid Singlet Oxygen Quencher. Archives of Biochemistry and Biophysics. 1989 November;274(2):532-538.

8. Chandra RV, Prabhuji MLV, D. Roopa DA, Ravirajan S, Kishore HC. Efficacy of Lycopene in the Treatment of Gingivitis: a Randomised, Placebo-controlled Clinical Trial. Oral Health Prev. Dent. 2007;5:327-336.

9. Wood N, Johnson RB. The relationship between tomato intake and congestive heart failure risk in periodontitis subjects. J Clin Periodontol. 2004;31:574-580.

10. Sharma A, Sharma S. Reactive Oxygen Species and Antioxidants in Periodontics: A Review. International Journal Of Dental Clinics 2011;3(2):44-47

11. Sree SL, Mythili R. Antioxidants in Periodontal Diseases: A Review. Indian Journal of Multidisciplinary Dentistry 2011 March-April;1(3):140-146.

12. Kutzing M, Firestein BL. Altered Uric Acid Levels and Disease States. J Pharmacol and Exp Therapeutics. 2008;324(1):1-7.

13. Nishida M, Grossi SG, Dunford RG, How A, Trezisan M, Genco RJ. Dietary vitamin $\mathrm{C}$ and the risk for periodontal disease, J Periodontol. 2000;71:1215-23.

14. Riso P, Pinder A, Santangelo A, Porrini M. Does tomato consumption effectively increase the resistance of lymphocyte DNA to oxidative damage. Am J Clin Nutr 1999; 69:712-718.

15. Panjamurthy K, Manoharan S, Ramachandran CR. Lipid peroxidation and antioxidant status in patients with periodontitis. Cell Mol Biol Lett 2005;10:255-264.

16. Sobaniec H, Sobaniec W, Sendrowski K, Sobaniec S, Pietruska M. Antioxidant activity of blood serum and saliva in patients with periodontal disease treated due to epilepsy. Advances in Medical Sciences 2007;52(Suppl. 1):204-206.

17. Akman S, Canakci V, Kara A, Tozoglu U, Arabaci T, and Dagsuyu IM. Therapeutic Effects of Alpha Lipoic Acid and Vitamin C on Alveolar Bone Resorption after Experimental Periodontitis in Rats: A Biochemical, Histochemical, and Stereologic Study. J Periodontol 2013;84:666-674.

18. Trivedi S, Nandla, Mahdi AA, Mittal M, Singh B, Pandey S. Evaluation of Antioxidant Enzymes Activity and Malondialdehyde Levels in Chronic Periodontitis Patients with Diabetes. J Periodontol 2014 May;85(5):713-20 